\title{
Flow and heat transfer of Oldroyd-B nanofluid with relaxation- retardation viscous dissipation and hyperbolic boundary conditions
}

\author{
S Mishra ${ }^{1}$, A Misra ${ }^{2}$, M K Nayak \\ ${ }^{1}$ Department of Mathematics, P N. Autonomous College, Utkal University, Bhubaneswar- \\ 752057, Odisha, India \\ ${ }^{2}$ Department of Mathematics, Centurian University of Technology and Management, \\ Paralakhemundi, Gajapati-761211, Odisha, India \\ ${ }^{3}$ Department of Physics, Radhakrishna Institute of Technology and Engineering, Bhubaneswar- \\ 752057, Odisha, India
}

Received: 6 March 2020; Received in revised form: 17 April 2020; Accepted: 22 April 2020; Published online April 292020

(C) Published at www.ijtf.org

\begin{abstract}
In the present research article, modeling and computations are presented to introduce the novel concept of relaxation-retardation viscous dissipation and hyperbolic time variation boundary conditions on the magnetohydrodynamic transient flow of Oldroyd-B nanofluid past a vertical stretched plate for the first time. In the present work, firstly we implement Buongiorno's model to illustrate Brownian motion and thermophoretic diffusion which take vital role in heat and mass transportation process. Nonlinear non-dimensional governing equations are solved by fourth order Runge-Kutta method along with shooting technique. We investigate the behavior of influential variables on the velocity, thermal and solutal fields through graphical illustrations. Our results indicate that relaxation and retardation Deborah numbers exhibit completely reverse trend in the flow field. Especially, augmented relaxationretardation viscous dissipation invigorates the temperature gradient. The results of the current theoretical study may be instrumental for worthful practical applications.
\end{abstract}

Keywords: Relaxation-retardation viscous dissipation; MHD; Unsteady flow; Oldroyd-B nanofluid; Hyperbolic time varying boundary conditions.

\section{Introduction}

Authors pay sincere thanks to the researchers for their precious contributions through fair, honest and expeditious investigations to the important progress achieved in the 
S. Mishra et al.

International Journal of Thermofluid Science and Technology (2020), Volume 7, Issue 1, Paper No. 20070104

accelerated research world over the past decades. Out of numerous dedicated contributions one remarkable achievement of Maxwell [1] is the development of Maxwell model.

\section{Nomenclature}

$(u, v)$ velocity components in $(\mathrm{x}, \mathrm{y})$ directions $\quad a>0$ is a constant

$u_{w}(x)$ plate velocity

$(T, C)$ fluid ( temperature, concentration)

$\left(T_{\infty}, C_{\infty}\right)$ ambient ( temperature, concentration) $\quad \alpha_{f} \quad$ thermal diffusivity

$\left(T_{w}, C_{w}\right)$ surface ( temperature, concentration) $\quad \rho_{f} \quad$ density of fluid

$F(\eta)$ dimensionless stream function $\quad \eta \quad$ non-dimensional vertical distance

$F_{\eta}(\eta)$ differentiation of $F(\eta)$ with respect to $\eta \quad \operatorname{Pr}=\frac{v}{\alpha}$ Prandtl number

$\left(\Gamma_{1}, \Gamma_{2}\right)$ relaxation time and retardation time $\quad \tau=\left(\rho C_{p}\right)_{p} /\left(\rho C_{p}\right)_{f} \quad$ heat capacity ratio

$N_{b}=\frac{\tau D_{B}\left(C_{w}-C_{\infty}\right)}{v_{f}}$ Brownian motion parameter $\quad M=\frac{\sigma B_{0}^{2}(1+\zeta t)}{a \rho_{f}}$ Hartmann number

$N_{t}=\frac{\tau D_{T}\left(T_{w}-T_{\infty}\right)}{T_{\infty} v_{f}} \quad$ thermophoresis parameter $\quad S c=\frac{v}{D_{B}}$ Scmidt number

$\Omega_{1}=\frac{a \Gamma_{1}}{1+\zeta t}$ Deborah number for relaxation time

$\Omega_{2}=\frac{a \Gamma_{2}}{1+\zeta t}$ Deborah number for retardation time

$E c_{m}=\frac{\Omega_{2} u_{w}^{2}}{\Omega_{1}\left(c_{p}\right)_{f}\left(T_{w}-T_{\infty}\right)}$ modified Eckert number

\section{Subscripts}

$f \quad$ fluid

w quantities at wall

$\infty \quad$ quantities at free stream

This is a rate type model used for evaluating the viscoelastic nature of materials. Ignoring the possibility of incompressible nature of liquid is another important feature of this model. It is indeed Oldroyd [2] after Maxwell, developed a constitutive relationship for describing actual behavior of nonlinear liquids. The constitutive relations of Oldroyd-B fluid characterizes the influence of both relaxation and retardation times. The Oldroyd-B model developed by Oldroyd becomes Maxwell model without retardation time. Many noteworthy researchers manoeuvred strenuous endeavors to study the flow and heat transfer of Oldroyd- 
S. Mishra et al.

International Journal of Thermofluid Science and Technology (2020), Volume 7, Issue 1, Paper No. 20070104

$\mathrm{B}$ fluid under different conditions and configurations. The outcomes of their investigations have been addressed and utilized by many others. Bhatnagar et al. [3] showed in their study how free stream velocity influences the flow of an Oldroyd-B fluid due to a stretching sheet. The effect of decay of a potential vortex in the flow of an Oldroyd-B fluid was investigated by Fetecau andFetecau [4]. The stagnation point flow of an Oldroyd-B fluid past a stretching sheet was studied intensively by Sajid et al. [5]. Zheng et al. [6] established exact solutions for MHD flow of generalized Oldroyd-B fluid due to an infinite accelerating plate. In their study, the fractional calculus approach is implemented to establish the constitutive relationship of the Oldroyd-B fluid. The solutions are developed by means of Fourier sine and Laplace transforms. Hayat et al. [7] examined three-dimensional convective flow of an Oldroyd-B fluid over a stretched surface. Their study addressed the convergence of series solutions by the homotopy analysis method (HAM). Abbasbandy et al. [8] analyzed the numerical and analytical solutions for Falkner-Skan flow of MHD Oldroyd-B fluid. The relaxation and retardation times have opposite effects on the velocity components were the results of their investigation. Interestingly, an unsteady helical flow of Oldroyd-B fluids was studied by Jamil et al. [9]. Oscillating motion of an Oldroyd-B fluid between two infinite circular cylinders was analyzed by Fetecau et al. [10]. Impact of Cattaneo-Christov heat flux in MHD flow of Oldroyd-B fluid with homogeneous-heterogeneous reactions was investigated by Hayat et al. [11]. Hayat et al. [12] applied non-Fourier heat flux theory to study on 2D stratified flow of an Oldroyd-B fluid with chemical reaction. They declared in their study that temperature distribution has opposite behavior for thermal relaxation time and variable thermal conductivity parameter. Simultaneous impacts of mixed convection and nonlinear thermal radiation in stagnation point transient flow of Oldroyd-B fluid was discussed by Hayat et al. [13]. They observed that the behavior of thermal relaxation and retardation times on velocity distribution are opposite. They also observed that temperature ratio and radiation parameters augment the temperature distribution. Zhang et al. [14] examined the flow and heat transfer of an Oldroyd-B nanofluid thin film over an unsteady stretching sheet. The influences of various relevant parameters such as unsteady parameter, volume fraction of $\mathrm{Cu} / \mathrm{Ag}$ and Prandtl number on the flow field were explored in their study.

Here we pronounce that the concept of nanofluid is developed by Choi [15] where the proper dilution and suspensions of the poor heat transfer fluids such as oil, water and ethylene glycol takes place with high thermal conductivity material nanoparticles like $\mathrm{Cu}, \mathrm{CuO}, \mathrm{Al}_{2} \mathrm{O}_{3}, \mathrm{TiO}_{2}, \mathrm{SiO}_{2}, \mathrm{ZrO}_{2}, \mathrm{ZnO}$. Nanofluid due to its unique and exceptional characteristic properties ensures a substantial upgradation of heat transfer of conventional (poor heat transfer) fluids. Consequently, such fluids has tremendously overwhelming the demand of heat removal in cooling applications. The most promising and inevitable diversified applications of nanofluids today include process industries, heat exchangers, cooling towers, transportation, magneto-optical devices, biomedical-therapeutic treatment and developing the best quality lubricants and oils and many others. Keeping the above astounding relevance into mind, many researchers succeeded in finding different models, phenomena and mechanisms theoretically and experimentally that would enhance the heat transfer capability of various nanofluids. Many authors have also studied the behavior of different nanofluids subject to several flow configurations and physical boundary conditions. Xuan and Li [16] studied the flow and convective heat transfer of nanofluids. Further, Buongiorno [17] introduced a two-phase model comprising the role of two slip mechanisms namely Brownian diffusion and thermophoresis which account for the thermal 
S. Mishra et al.

International Journal of Thermofluid Science and Technology (2020), Volume 7, Issue 1, Paper No. 20070104

conductivity enhancement of nanofluids. Khan and Pop [18] analyzed the boundary-layer stretched flow of a nanofluid.

Moreover, series solutions for Oldroyd-B fluid motion induced by a deforming sheet having exponential velocity were investigated by Hayat et al. [19]. In their study, they considered the wall temperature as exponentially growing function of the horizontal distance. Shivakumara et al. [20] analyzed thermal convective instability in an Oldroyd-B nanofluid embedded in porous layer. In their analysis, the authors implemented the famous Buongiorno model and assumed zero nanoparticle flux at the boundaries. Nayak [21] examined the impact of thermal radiation and viscous dissipation on three dimensional magnetohydrodynamic flow nanofluids by shrinking surface using Homotopy analysis method (HAM). It seen that there is an increase in temperature due to an increase in thermal radiation parameter leads to lower heat transfer rate from the surface of the sheet. Also it is noticed from his study that the local Nusselt number gets reduced indicating lowering heat transfer rate from the surface with increasing Eckert number. Nayak et al. [22] explored the effects of velocity slip and non-linear thermal radiation on three dimensional magnetohydrodynamic convective flows of nanofluids through porous media. Increasing values of slip parameter slow down the axial and transverse fluid velocities and that of temperature parameter yielding greater non-linearity in heat transfer rate from the surface are important outcomes of their investigation. Nayak et al. [23] studied the effect of thermal radiation and natural convection of three dimensional magnetohydrodynamic flows of nanofluids over permeable linear stretching sheet. They declared in their study that rising the buoyancy and radiation parameter uplift the velocity and temperature profiles respectively. Ghadikolaei et al. [24] showed in his study how the porous matrix and thermal radiation influences significantly on flow and heat transfer of $\mathrm{Fe}_{3} \mathrm{O}_{4}-\left(\mathrm{CH}_{2} \mathrm{OH}\right)_{2}$ nanofluids. Hayat et al. [25] revealed that the heat generation parameter upgrades the fluid temperature and the related thermal boundary layer in the stretched flow of magnetohydrodynamic Oldroyd-B nanofluid. The flow of magnetized Oldroyd-B fluid over a rotating disk influenced by nonlinear radiation and activation energy was analyzed by Khan et al. [15]. In their study, they developed numerical solution and conveyed that temperature ratio parameter augments the temperature distributions in the boundary layer region. Mahanthesh et al. [27] discussed the nonlinear three-dimensional stretched flow of an Oldroyd-B fluid with convective condition. Kumar et al. [28] investigated the effect of Joule heating and viscous dissipation on threedimensional flow of Oldroyd B nanofluid with thermal radiation where they confirmed that rise in Eckert number led to the escalation of temperature fields in the entire flow domain.

Viscous dissipation finds convenience to take place in stronger gravitational fields, larger planets, heavier gases in space and geological operations. Viscous dissipation is a partial irreversible process that generates an additional heat in the flow process due to fluid friction. Nayak [29] explored the nature of dissipative flow of nanofluids under the influence of transverse magnetic field in association with thermal radiation embedded in a porous medium. It is seen in his study that an increase in Eckert number increases the fluid temperature and the associated thermal boundary layer thickness. This leads to reduction of the rate of heat transfer from the stretched surface. This reduction is further decreased due to presence of porous medium. Therefore, the presence of porous medium acts as an insulator to the surface of the sheet.

Going through the aforementioned literature survey, it is well understood that until now there is only one publication about the aspect of relaxation-retardation viscous dissipation. Only Zhang et al. [30] studied the behavior of relaxation-retardation viscous 


\section{S. Mishra et al.}

International Journal of Thermofluid Science and Technology (2020), Volume 7, Issue 1, Paper No. 20070104

dissipation of Oldroyd-B fluid thin film. However, the impact of relaxation-retardation viscous dissipation on the flow of Oldroyd-B nanofluid past a stretched vertical plate associated with hyperbolic time varying boundary conditions has not yet studied. The aim of this study is to bridge such gap. The prime objective of our research is to model the Oldroyd-B nanofluid flow subject to relaxation-retardation viscous dissipation past a stretched vertical plate associated with hyperbolic time varying boundary conditions. Heat and mass transfer process are explored due to Buongiorno's model featured by aspects of Brownian motion and thermophoretic diffusion. The fourth order R-K method along with shooting technique is adopted as a numerical tool to solve the resulting nonlinear governing expressions. The characteristics of velocity, thermal and solutal distributions, surface drag force, heat and mass transfer rates well displayed and discussed.

\section{Formulation of the problem}

In In this problem we deal with the time-varying flow of Oldroyd B nanofluid past a stretched vertical plate. Two familiar phenomena such as thermophoretic motion and Brownian diffusion have been taken into account. Relaxation-retardation viscous dissipation is introduced. Hyperbolic time varying boundary conditions are implemented. Assume that $u$ and $v$ are respectively the velocity components in $\mathrm{x}$ - and $\mathrm{y}$ - directions where $\mathrm{x}$-axis is along the plate and y-axis is at right angle to it as represented in Fig.1.

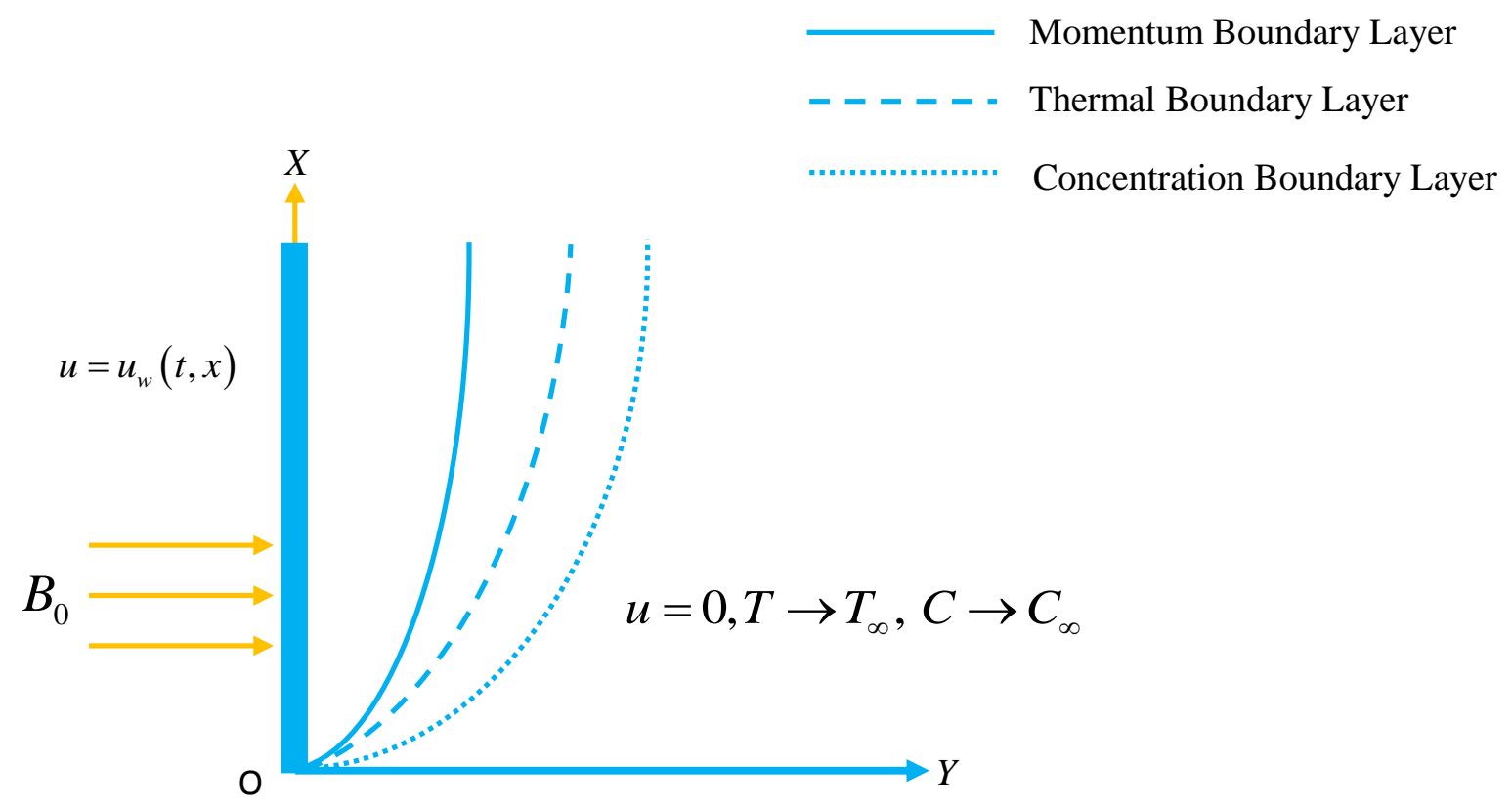

Fig. 1 Flow geometry and the associated coordinate system of the problem. 
S. Mishra et al.

International Journal of Thermofluid Science and Technology (2020), Volume 7, Issue 1, Paper No. 20070104

The underlying boundary layer equations with the very idea of Boussinesq approximation and above declared assumptions are [3], [18], [26-28], [30]:

$$
\begin{aligned}
& u_{x}+v_{y}=0 \\
& u_{t}+u u_{x}+v u_{y}+\Gamma_{1}\left(u^{2} u_{x x}+v^{2} u_{y y}+2 u v u_{x y}\right) \\
& =v_{f}\left[u_{y y}+\Gamma_{2}\left(u u_{x y y}+v u_{y y y}-u_{x} u_{y y}-u_{y} v_{y y}\right)\right]-\frac{\sigma B_{0}^{2} u}{\rho_{f}} \\
& T_{t}+u T_{x}+v T_{y}=\alpha_{f} T_{y y}+\tau\left[D_{B} C_{y} T_{y}+\frac{D_{T}}{T_{\infty}}\left(T_{y}^{2}\right)\right]+\frac{\Gamma_{2}}{\Gamma_{1}} \frac{\mu_{f}}{\left(\rho C_{p}\right)_{f}}\left(u_{y}^{2}\right) \\
& C_{t}+u C_{x}+v C_{y}=D_{B} C_{y y}+\frac{D_{T}}{T_{\infty}} T_{y y}
\end{aligned}
$$

The required hyperbolic time variation boundary conditions are:

$$
\left.\begin{array}{rrr}
\text { At } t=0, & u=v=0, T=T_{\infty}, C=C_{\infty} \quad(x, y \geq 0) \\
u=u_{w}(t, x), v=0, T=T_{w}, C=C_{w} & (y=0) \\
\text { At } t>0, & u=0, T \rightarrow T_{\infty}, C \rightarrow C_{\infty} \quad \text { as } \quad y \rightarrow \infty
\end{array}\right\}
$$

where

$$
u_{w}=\frac{a x}{1+\zeta t}
$$

$$
\left.T_{w}=T_{\infty}+\frac{T_{0} x^{m}}{1+\zeta t}, C_{w}=C_{\infty}+\frac{C_{0} x^{n}}{1+\zeta t}\right\}
$$

Here, $u$ and $v$ are the fluid velocity components along $\mathrm{x}$ and $\mathrm{y}$ directions, $u_{w}(x)$ is the plate velocity, $a>0$ is a constant.

The appropriate transformations used are

$$
\left.\begin{array}{c}
u=\frac{a x}{1+\zeta t} F_{\eta}(\eta), v=-\sqrt{\frac{a v_{f}}{1+\zeta t}} F(\eta), \\
\theta(\eta)=\frac{T-T_{\infty}}{T_{w}-T_{\infty}}, \phi(\eta)=\frac{C-C_{\infty}}{C_{w}-C_{\infty}}, \eta=y \sqrt{\left(\frac{a}{v_{f}(1+\zeta t)}\right)}
\end{array}\right\}
$$

where $F_{\eta}(\eta)$ is the differentiation with respect to $\eta$.

Using eqs (6) and (7), eqs (2-5) take the form

$$
\begin{aligned}
& F_{\eta \eta \eta}+\Omega_{2}\left[\left(F_{\eta \eta}\right)^{2}-F F_{\eta \eta \eta \eta}\right]+\Omega_{1}\left(2 F F_{\eta} F_{\eta \eta}-F^{2} F_{\eta \eta \eta}\right) \\
& +F F_{\eta \eta}-\left(F_{\eta}\right)^{2}+\frac{1}{2} \varepsilon \eta F_{\eta \eta}+\varepsilon F_{\eta}-M F^{\prime}=0 \\
& \theta_{\eta \eta}+\operatorname{Pr}\left[N_{b} \theta_{\eta} \phi_{\eta}+N_{t}\left(\theta_{\eta}\right)^{2}+F \theta_{\eta}+\frac{1}{2} \varepsilon \eta \theta_{\eta}+2 \operatorname{Pr} E_{c m}\left(F_{\eta \eta}\right)^{2}\right]=0
\end{aligned}
$$


$\phi_{\eta \eta}+\left(\frac{N_{b}}{N_{t}}\right) \theta_{\eta \eta}-S c\left(F \phi_{\eta}+\frac{1}{2} \varepsilon \phi_{\eta}\right)=0$

with

$$
\left.\begin{array}{c}
F_{\eta}=1, F=0, \theta=1, \phi=1 \text { at } \eta=0 \\
F_{\eta}=0, \theta \rightarrow 0, \phi \rightarrow 0 \text { as } \eta \rightarrow \infty
\end{array}\right\}
$$

Here

$$
\left.\begin{array}{c}
\Omega_{1}=\frac{a \Gamma_{1}}{1+\zeta t}, \Omega_{2}=\frac{a \Gamma_{2}}{1+\zeta t}, \varepsilon=\frac{\zeta}{a}, \\
M=\frac{\sigma B_{0}^{2}(1+\zeta t)}{a \rho_{f}}, N_{t}=\frac{\tau D_{T}\left(T_{w}-T_{\infty}\right)}{T_{\infty} v_{f}}, \operatorname{Pr}=\frac{v}{\alpha} \\
S c=\frac{v}{D_{B}}, E c_{m}=\frac{\Omega_{2} u_{w}^{2}}{\Omega_{1}\left(c_{p}\right)_{f}\left(T_{w}-T_{\infty}\right)}, N_{b}=\frac{\tau D_{B}\left(C_{w}-C_{\infty}\right)}{v_{f}}
\end{array}\right\}
$$

The local skin friction coefficient is written as

$$
C_{f x}=\frac{\tau_{w}}{\frac{1}{2} \rho u_{w}^{2}}
$$

where $\tau_{w}=\mu\left(\frac{\partial u}{\partial y}\right)_{y=0}$ is the wall shear stress.

The non dimensional local skin friction coefficient is

$$
\operatorname{Re}_{x}^{\frac{1}{2}} C_{f x}=\frac{1}{2} \sqrt{a} F_{\eta \eta}(0)
$$

The local Nusselt and Sherwood numbers are respectively

$$
N u_{x}=\frac{x q_{w}}{k_{f}\left(T_{f}-T_{\infty}\right)}
$$

and

$$
S h_{x}=\frac{x j_{w}}{D_{B}\left(C_{w}-C_{\infty}\right)}
$$

where $q_{w}=-k_{f}\left(\frac{\partial T}{\partial y}\right)_{y=0}$ and $j_{w}=-D_{B}\left(\frac{\partial C}{\partial y}\right)_{y=0}$ are the wall heat and concentration flux respectively .

The non-dimensional local Nusselt and Sherwood numbers can be developed as

$$
\begin{aligned}
& R e_{x}^{-1 / 2} N u_{x}=-\theta_{\eta}(0) \\
& R e_{x}^{-1 / 2} S h_{x}=-\phi_{\eta}(0)
\end{aligned}
$$

and

where $\operatorname{Re}_{x}=\frac{x u_{w}(x)}{v_{f}}$ is the local Reynolds number. 
S. Mishra et al.

International Journal of Thermofluid Science and Technology (2020), Volume 7, Issue 1, Paper No. 20070104

\section{Solution method and validation}

In the present study, the nonlinear and coupled Eqs (8), (9), and (10) with boundary conditions (11) are solved numerically using Runge-Kutta-Fehlberg method with shooting technique for different values of parameters. Table 1 conveys the comparison of present results with the previously declared results of Abel et al. [33], Megahed [34] and Waqas et al. [35] where it is visualized that there is well and good agreement between them. The good agreement is related to the visualization that augmentation of Deborah number $\Omega_{1}$ associated with relaxation time upsurges the skin friction significantly.

\section{Results and Discussion}

This section presents a bold and visionary analysis providing the physical interpretation due to the interaction of interesting assorted variables including Deborah numbers $\left(\Omega_{1}\right.$ and $\left.\Omega_{2}\right)$, Hartmann number $M$, Prandtl number $\operatorname{Pr}$, modified Eckert number $E_{c m}$, Schmidt number $S c$ with flow, thermal and solutal fields. The detailed information regarding behaviors of velocity, temperature and concentration fields, viscous drag, Heat transfer rate and rate of mass transfer associated with the time-varying flow of Oldroyd-B nanofluid past a stretched vertical plate influenced by relaxation-retardation viscous dissipation have been well addressed.

\subsection{Velocity distribution}

The observation of Fig. 2 indicates that controlled fluid motion is accomplished due to enhancement of Deborah number $\Omega_{1}$ associated with relaxation time. However, the adverse nature of fluid motion in response to Deborah number $\Omega_{2}$ associated with retardation time is visible in Fig. 3. From Figs. 2and 3 it is inferred that behavior of Deborah number $\Omega_{2}$ is quite opposite to that of $\Omega_{1}$. In fact, for higher relaxation time the Oldroyd nanofluid boosts up thereby responsible for the decline of the motion of the Oldroyd nanofluid. On the other hand, $\Omega_{2}$ corresponds to retardation time $\Gamma_{2}$. Physically, an augmentation in retardation time upsurges the flow. It is well agreed with the observation in Mustafa et al. [31]. Fig. 4 has rightly stated that increase in Hartmann number $M$ contributes decelerated motion and the related shrinking velocity boundary layer. Please bear in mind that the flow over the plate is restrained by Lorentz force which is generated due to the interaction between applied magnetic field and the conducting fluid.

\subsection{Temperature distribution}

The outcomes of the present analysis reveal many interesting aspects of the heat transfer in a flow of Oldroyd-B nanofluid under the impact of various pertinent thermal parameters. We start with Fig. 5 describes that fluid with high Prandtl number accounts for abatement of fluid temperature $\theta(\eta)$ and upgradation of heat transfer from the plate. The basic point is that Prandtl number has a reverse association with thermal diffusivity. So it is not surprised that for high Prandtl fluid (having larger value of $\operatorname{Pr}$ ) momentum diffusivity dominates over thermal diffusivity which responds to decaying of temperature. This is an excellent agreement with Hayat et al. [32]. 
S. Mishra et al.

International Journal of Thermofluid Science and Technology (2020), Volume 7, Issue 1, Paper No. 20070104

Fluid temperature $\theta(\eta)$ and the related layer thickness eventually develop the ascending trend in response to rising modified Eckert number $E c_{m}$ (portrayed in Fig. 6). In fact, increasing $E c_{m}$ contributes stronger viscous dissipation associated with the converted internal energy, which in turn boosts the temperature. Further, increase in Hartmann number $M$ (visualized in Fig. 7) causes establishment and development of escalated non-dimensional fluid temperature $\theta(\eta)$ and the related thermal - layer thickness. In fact, greater Lorentz force due to larger $M$ produces restrained fluid flow which in turn builds up more heat in the boundary layer.

\subsection{Concentration distribution}

Fig. 8 envisages that nanoparticles concentration $\phi(\eta)$ and the associated layer thickness undermine due to increasing Schmidt number $S c$. Higher $S c$ indicating lower molecular diffusivity contributes lesser nanoparticles concentration in the related boundary layer. At certain $S c$ (for instance, $S c=5), \phi(\eta)$ is maximum in the flow zone contiguous to the solid boundary and then $\phi(\eta)$ gradually diminishes as we proceed towards the ambient fluid.

\subsection{Local skin friction, Nusselt number and Sherwood number}

Fig. 9 reveals the surface drag force $F_{\eta \eta}(0)$ behavior or the skin friction characteristics in response to Deborah numbers $\Omega_{1}$ and $\Omega_{2}$ for different Hartmann number $M$. In this case $\Omega_{1}$ and $\Omega_{2}$ influence the wall shear stress in diagonally opposite manner. In this case, surface drag force slows down with increase in $\Omega_{1}$ while it escalates with rising $\Omega_{2}$ (opposite behavior is noticed). The skin friction is a decreasing function of $\Omega_{1}$ while it is an increasing function of $\Omega_{2}$. Here we must note that these two Deborah numbers exhibit exactly opposite nature of fluid velocity. This is well observed in Mustafa et al. [31]. Fig. 10 narrates how the Nusselt number varies in response to thermophoretic parameter $N_{t}$ and Brownian motion parameter $N_{b}$ against different values of Hartmann number $M$. A reduction in the absolute value of Nusselt number is accomplished due to elevating both $N_{t}$ and $N_{b}$. This implicates that heat transfer rate gets diminution due to the influence of Brownian motion and thermophoresis mechanism. However, descending trend is more eye-catching due to the influence of Brownian motion. In fact, rise in thermophoresis parameter $N_{t}$ improves the temperature profiles and the related layer thickness. As indicated in different literature, larger thermophoresis parameter indicates stronger thermophoretic force. Stronger thermophoretic force drags enormous nanoparticles through greater diffusion from the hot plate towards the ambient thereby increases the fluid temperature within the domain. An increase in $N_{b}$ corresponds to the effective random motion of fluid particles generating more heat within the flow. Therefore temperature increases. Fig. 11 delineates how the Sherwood number gets affected from the impact of $N_{t}$ and $N_{b}$ against different $M$. As a first step, by increasing $N_{t}$ leads to a diminishing trend of mass transfer rate while that of $N_{b}$ yields a reverse trend. This is because one can observe from diversified literature that increase in thermophoretic parameter $N_{t}$ witnesses an up gradation of nanoparticles concentration while augmented Brownian motion parameter $N_{b}$ shows a reverse trend. This is due to the fact that rising of $N t$ indicating strong thermophoretic force leading to larger diffusion of nanoparticles from the hot plate to the ambient fluid and as a result 
S. Mishra et al.

International Journal of Thermofluid Science and Technology (2020), Volume 7, Issue 1, Paper No. 20070104

improves nanoparticles concentration profiles. On the other hand, the Brownian force due to Brownian motion makes the particles to move in opposite direction of the concentration gradient and makes the nanofluid more homogenous. Such force leads to low concentration gradient and more uniform nanoparticle concentration distribution. It is interesting to note that thermophoretic force strength due to $N_{t}=0.6$ and Brownian force strength due to $N_{b}=0.7$ contribute almost same mass transportation in the associated boundary layer.

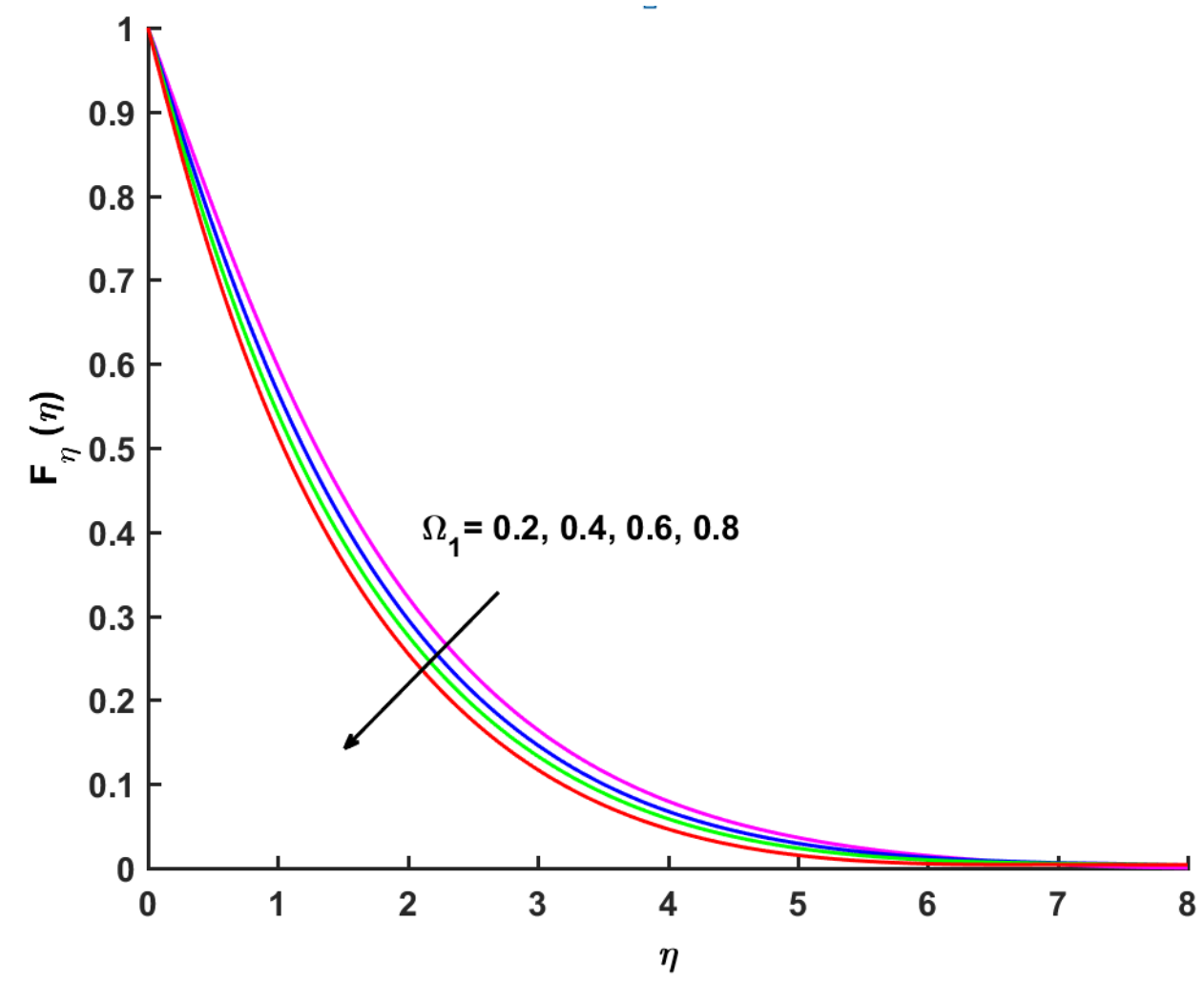

Fig. 2 Influence of Deborah number for relaxation time $\Omega_{1}$ on velocity $F_{\eta}(\eta)$ 


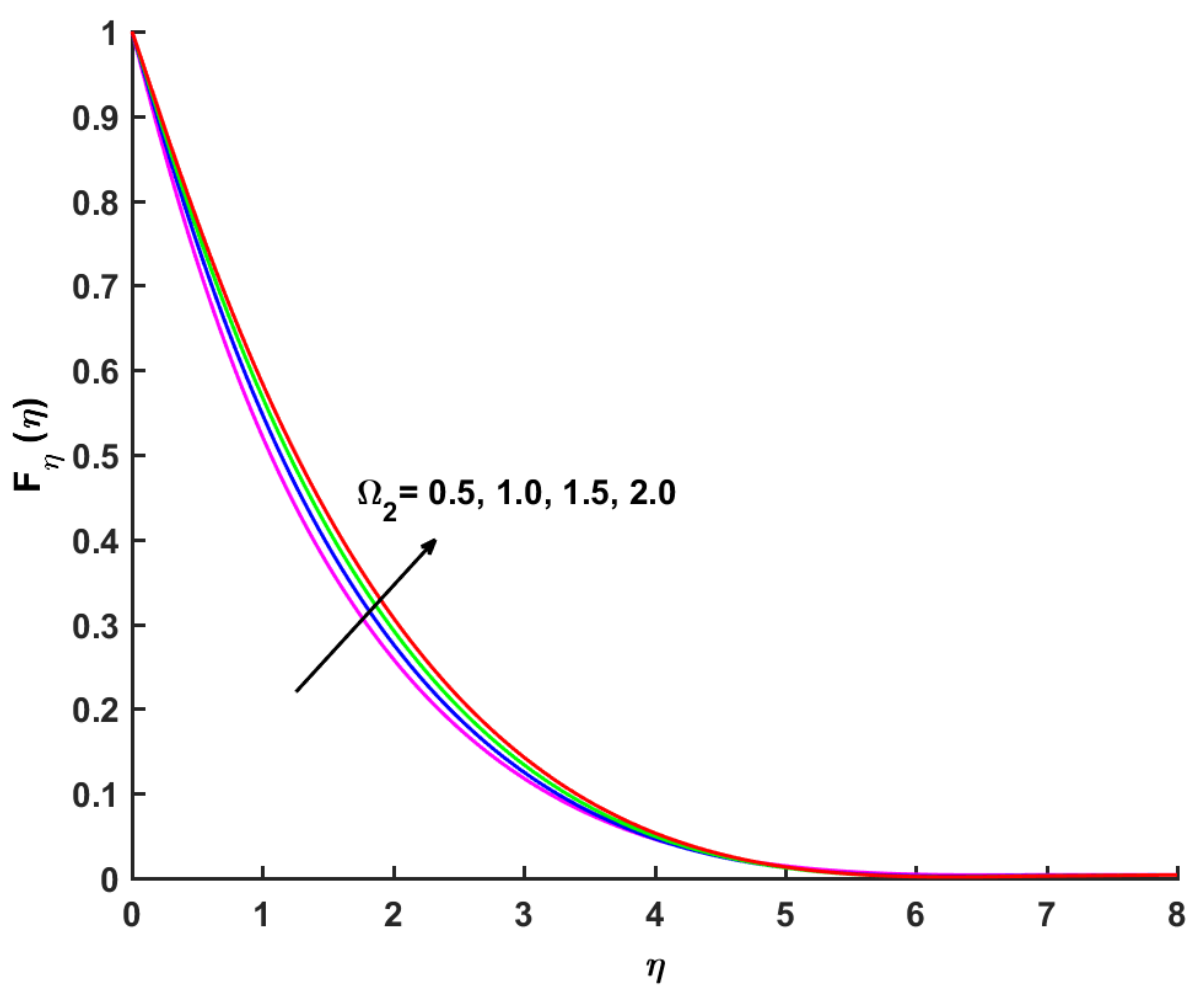

Fig. 3 Influence of Deborah number for retardation time $\Omega_{2}$ on velocity $F_{\eta}(\eta)$

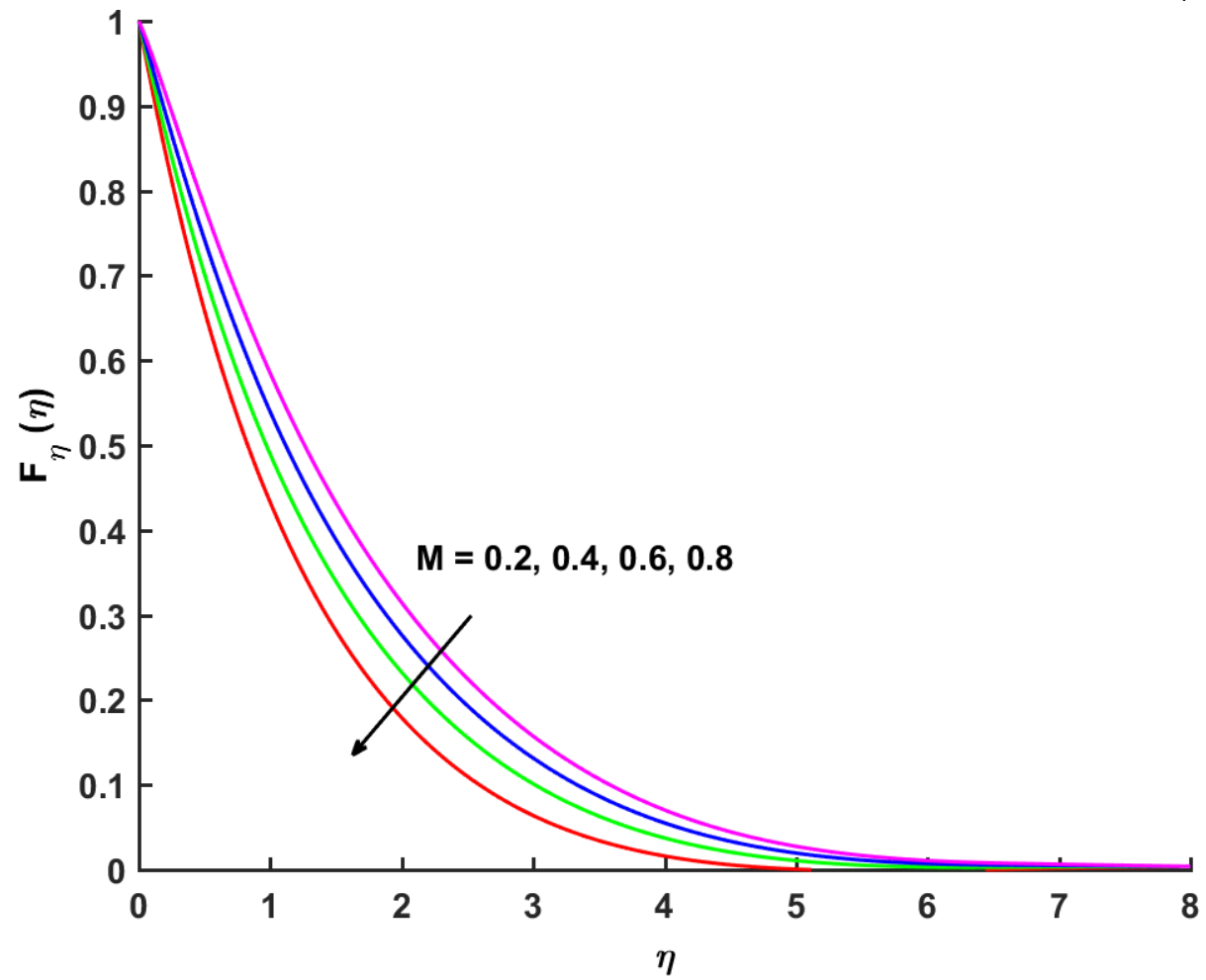

Fig. 4 Influence of Hartmann number $M$ on $F_{\eta}(\eta)$ 


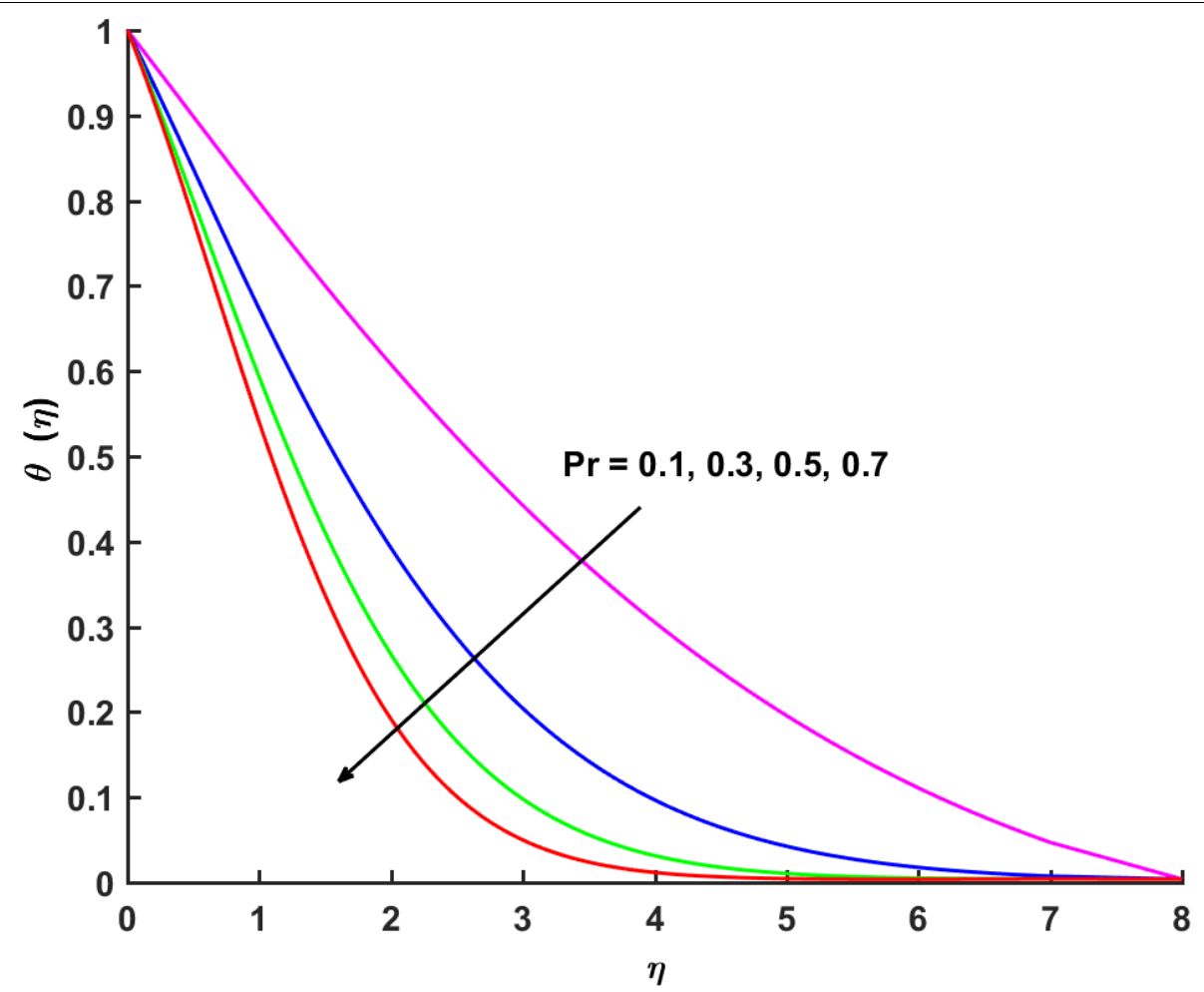

Fig. 5 Influence of Prandtl number Pr on temperature $\theta(\eta)$

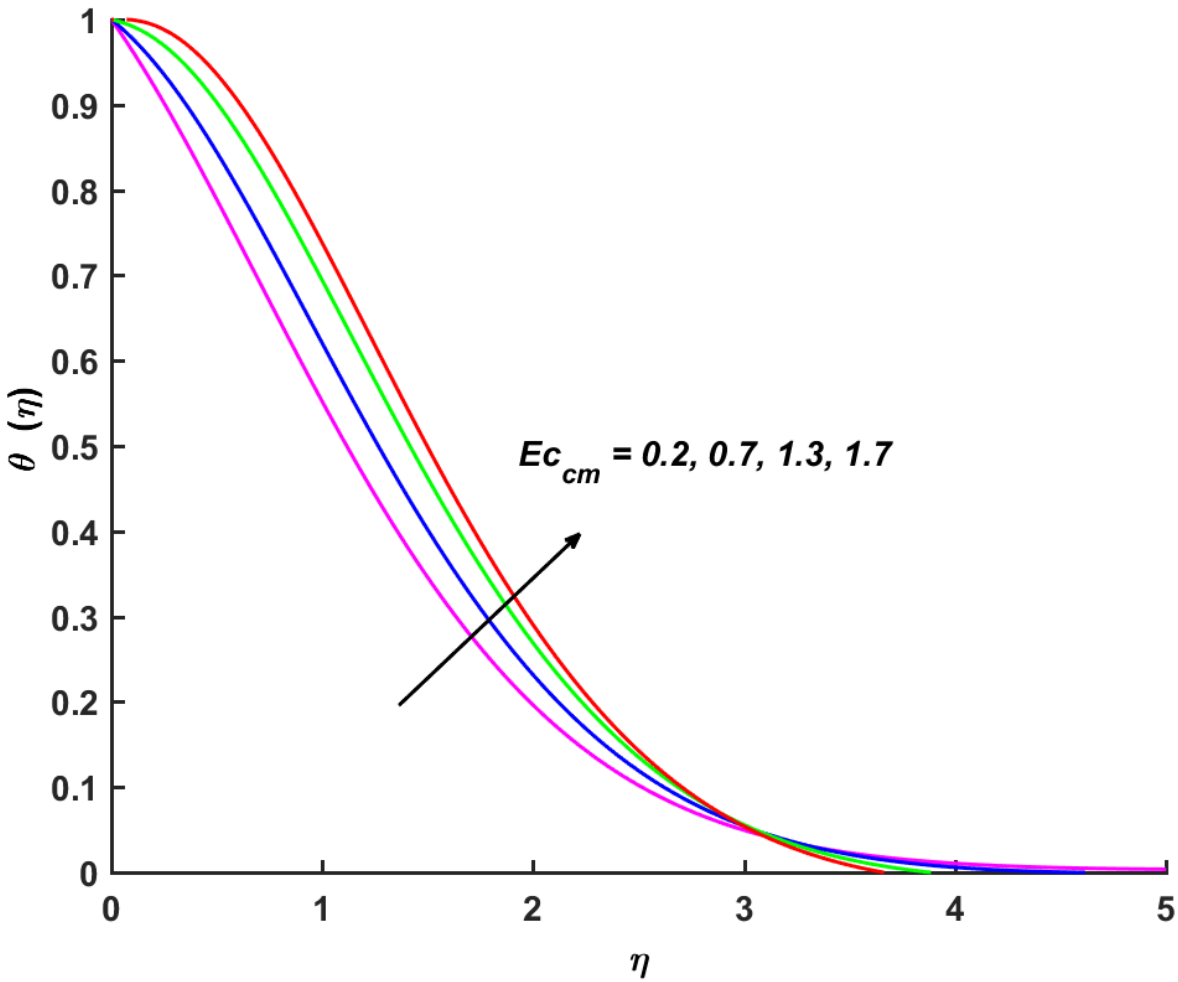

Fig. 6 Inflence of modified Eckert number $E c_{m}$ on $\theta(\eta)$ 


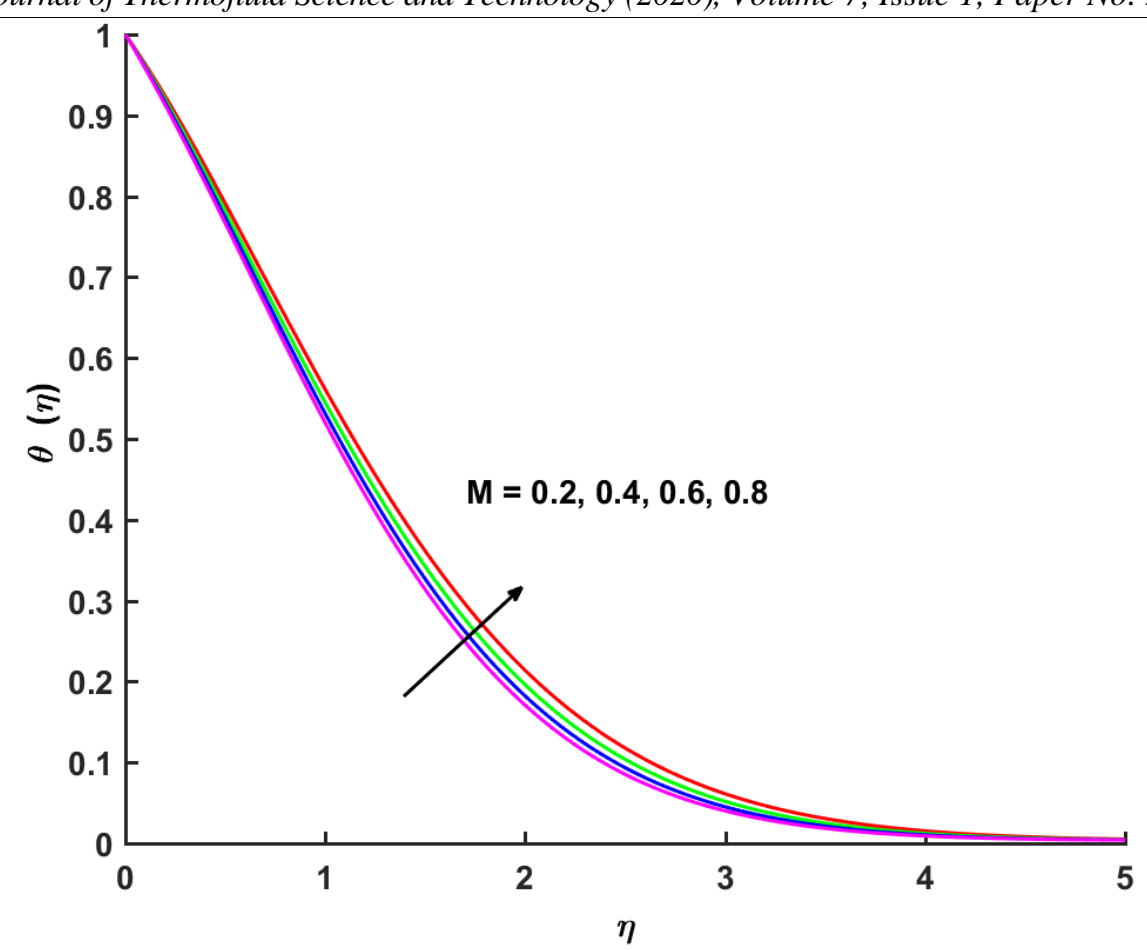

Fig.7 Influence of Hartmann number $M$ on $\theta(\eta)$

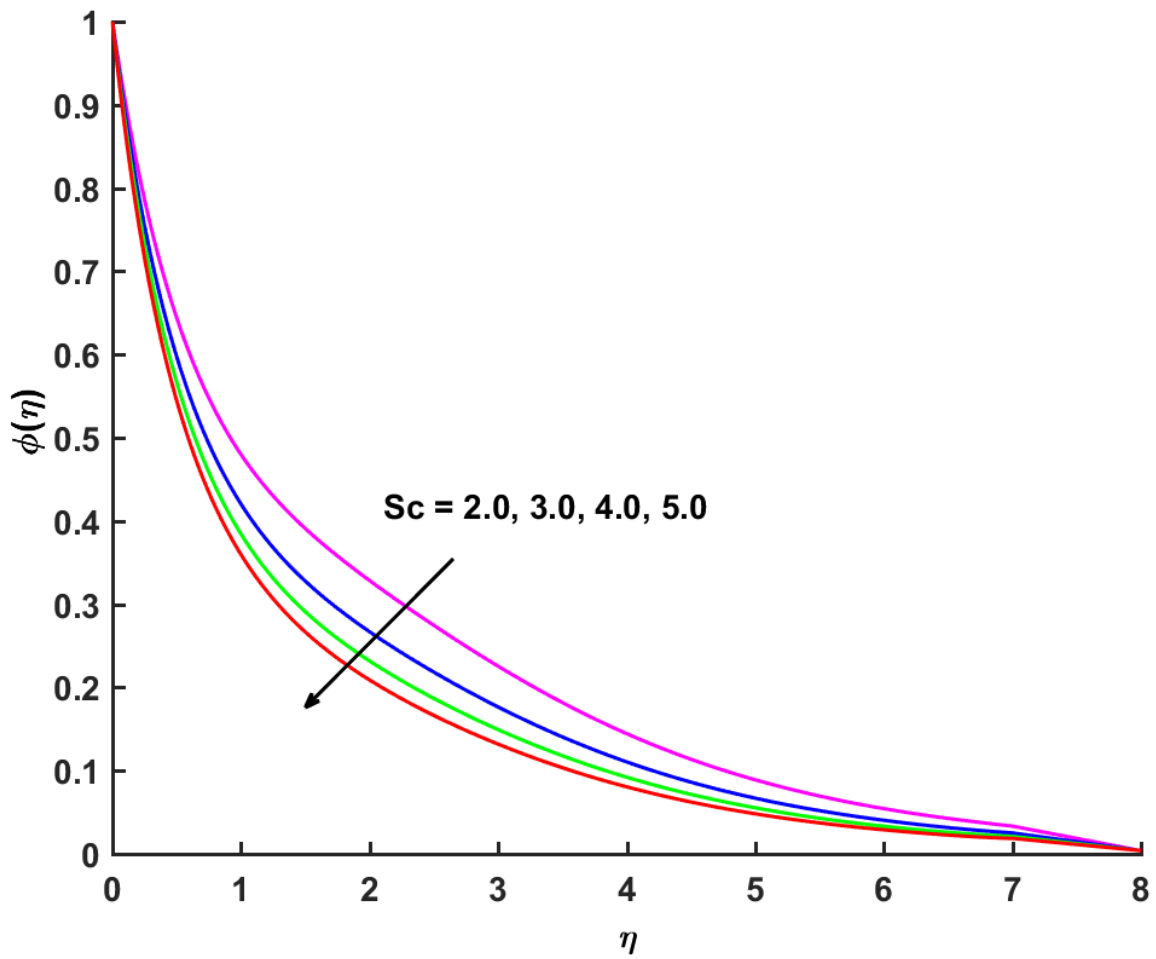

Fig. 8 Influence of Scmidt number $S c$ on $\phi(\eta)$ 


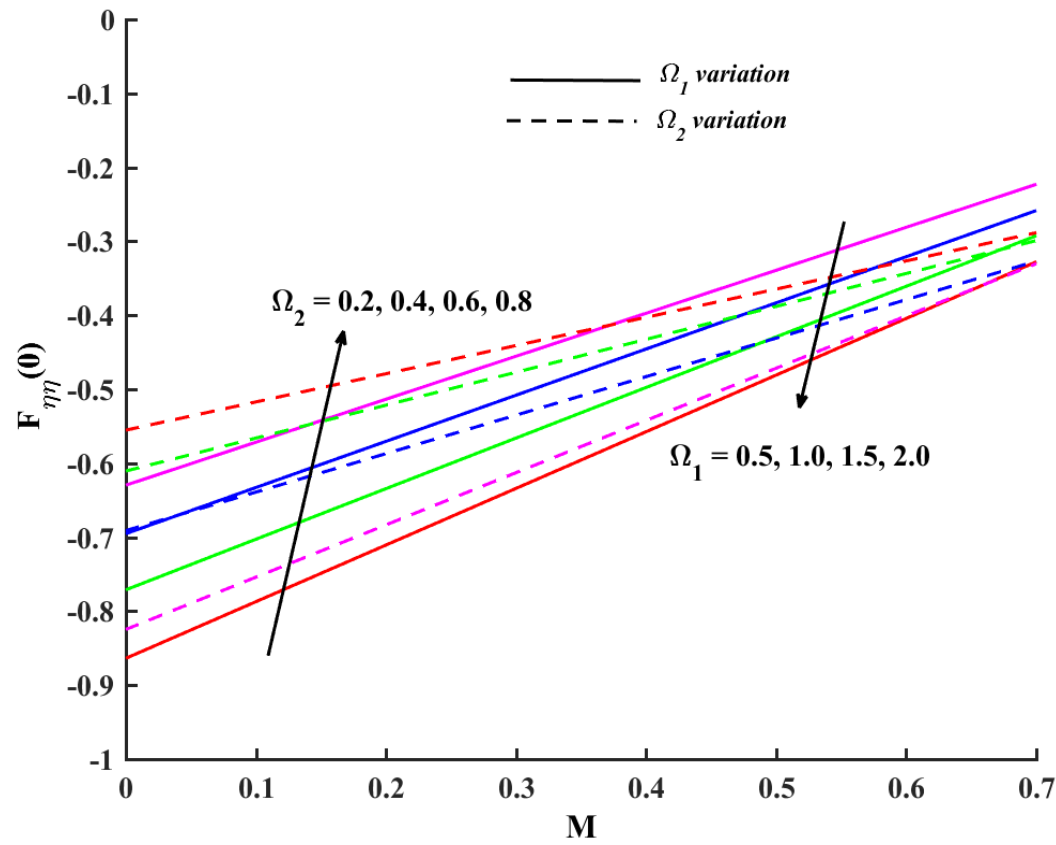

Fig. 9 Influence of $\Omega_{1}$ and $\Omega_{2}$ on skin friction against $M$

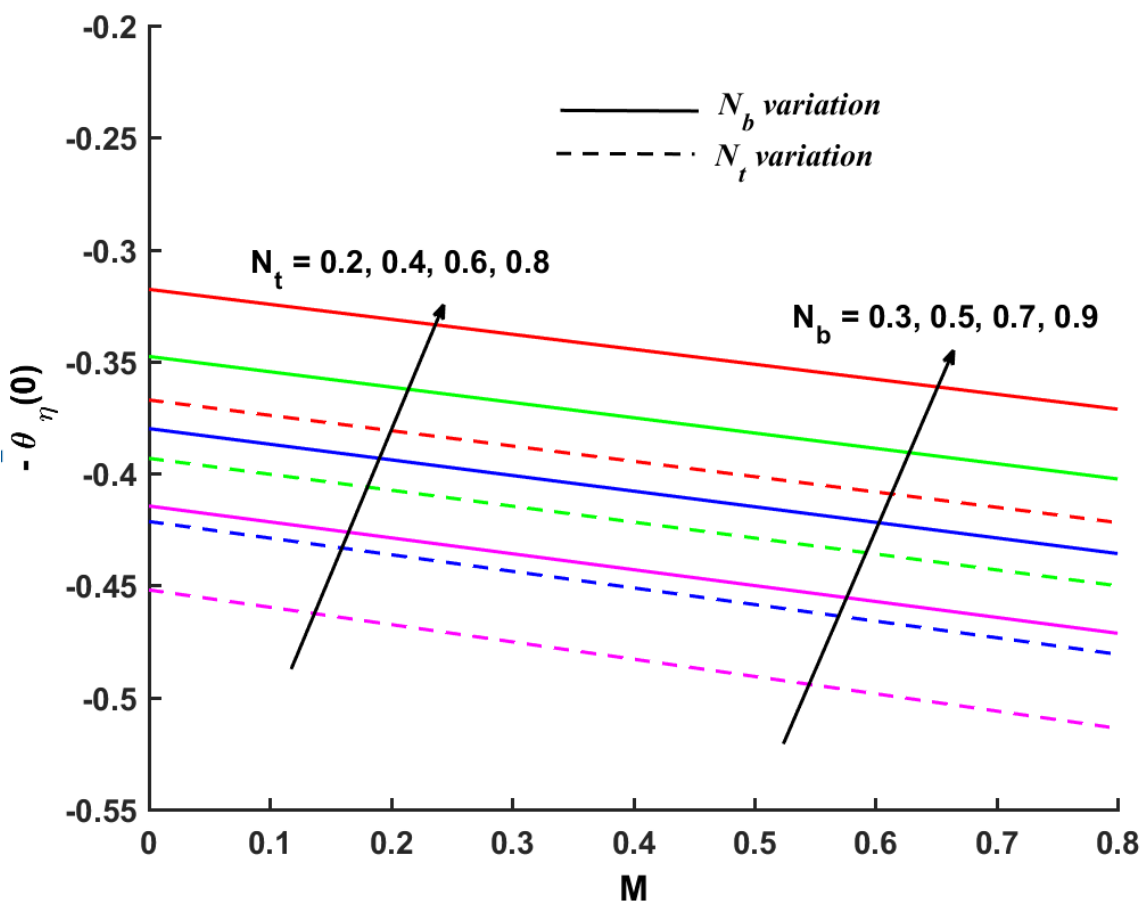

Fig. 10 Influence of $N_{t}$ and $N_{b}$ on Nusselt number against $M$ 


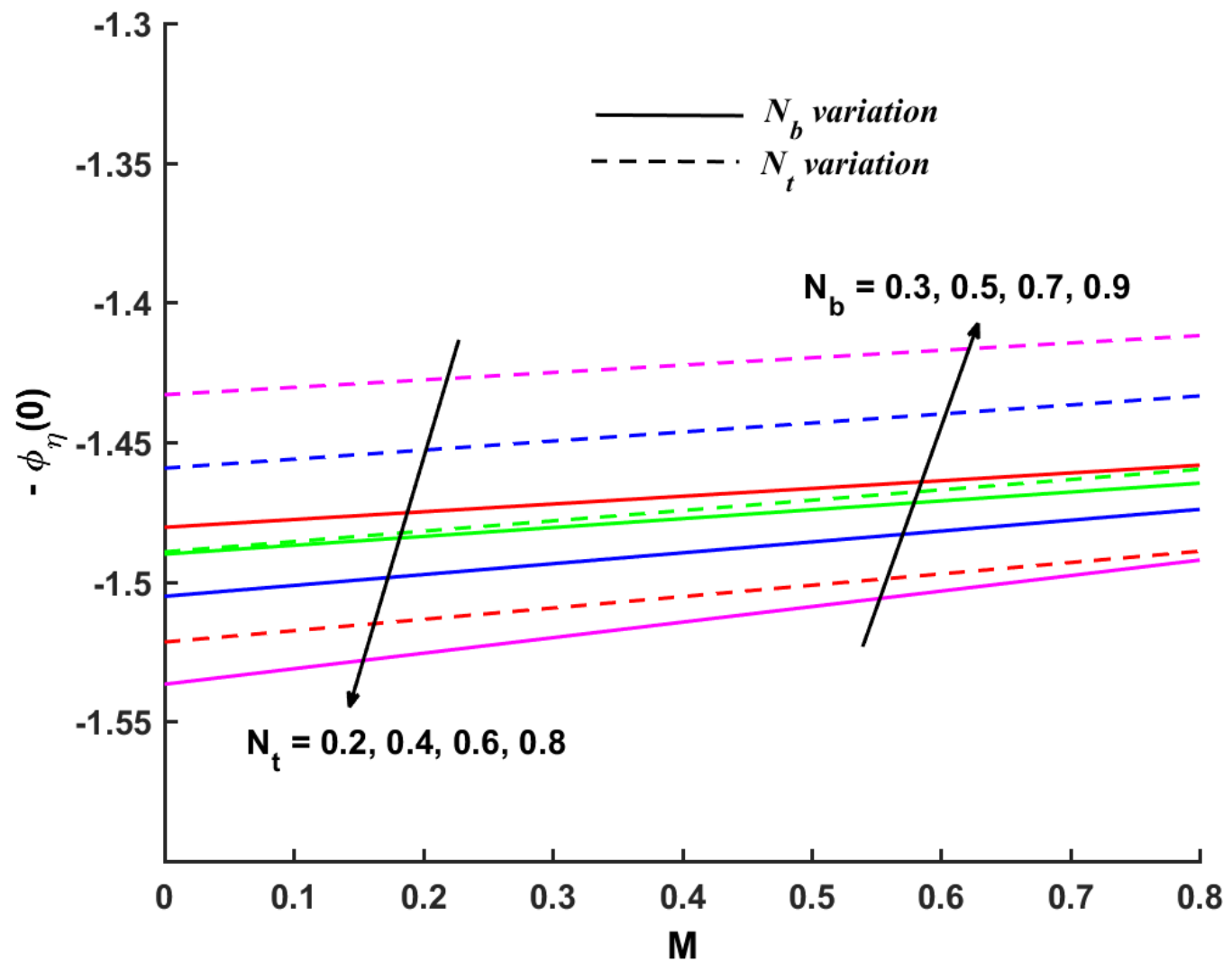

Fig. 11 Influence of $N_{t}$ and $N_{b}$ on Sherwood number against $M$

Table1. Comparison of $F_{\eta \eta}(0)$ for different values of $\boldsymbol{\Omega}_{\mathbf{1}}$ for $\boldsymbol{\Omega}_{\mathbf{2}}=\boldsymbol{\epsilon}=\boldsymbol{Z}=\boldsymbol{\delta}=\mathbf{0}$.

\begin{tabular}{|c|c|c|c|c|}
\hline $\mathbf{\Omega}_{\mathbf{1}}$ & Abel et al. [33] & Megahed [34] & Waqas [35] & Present Work \\
\hline 0.0 & 1.000000 & 0.999978 & 1.000000 & 1.00000000 \\
\hline 0.2 & 1.051948 & 1.051945 & 1.051889 & 1.05188988 \\
\hline 0.4 & 1.101850 & 1.101848 & 1.101903 & 1.10185163 \\
\hline 0.6 & 1.150163 & 1.150160 & 1.150137 & 1.15016093 \\
\hline 0.8 & 1.196692 & 1.196690 & 1.196711 & 1.19669083 \\
\hline 1.2 & 1.285257 & 1.285253 & 1.285363 & 1.28525740 \\
\hline 1.6 & 1.368641 & 1.368641 & 1.368758 & 1.36878678 \\
\hline 2.0 & 1.447617 & 1.447616 & 1.447651 & 1.44761469 \\
\hline
\end{tabular}

\section{Conclusion}

There have been reports regarding the flow and heat transfer of Oldroyd-B nanofluid in view of relaxation-retardation viscous dissipation over a vertical stretched plate. This convenient research grows our insights into innumerable significant practical applications, for example production of plastic sheets and extrusion of polymer in polymer industry. 
S. Mishra et al.

International Journal of Thermofluid Science and Technology (2020), Volume 7, Issue 1, Paper No. 20070104

Enhancement of Deborah numbers $\Omega_{1}$ and $\Omega_{2}$ associated with relaxation time accounts for diminishing trend of fluid motion while that of $\Omega_{2}$ (Deborah number associated with retardation number) exhibit adverse behavior. More Hartmann number $M$ indicating more Lorentz force yields diminutive fluid flow and escalates the fluid temperature. Deborah numbers $\Omega_{1}$ and $\Omega_{2}$ influence the wall shear stress in diagonally opposite manner for different Hartmann number $M$. Increasing thermophoretic force causes a diminution of mass transfer rate while a reverse situation is noticed for that of Brownian motion. Smaller thermophoretic force ( $\operatorname{small} N_{t}$ ) and less Brownian motion subject to considerably high Prandtl fluids lead to the enhancement of heat transfer rate from the vertical stretched plate.

At last, it has been suggested that the present model can be adopted for entropy optimized three dimensional rotating flow problems, which will be a focus in our future work.

\section{References}

[1] J. C. Maxwell, On the dynamical theory of gases, Philos. Trans. R. Soc. 157 (1867) 49- 88.

[2] J. G. Oldroyd, On the formulation of rheological equations of state, Proc. R. Soc. Lond. Ser. A 200 (1950) 523-541.

[3] R. K. Bhatnagar, G. Gupta and K. R. Rajagopal, Flow of an Oldroyd-B fluid due to a stretching sheet in the presence of a free stream velocity, Int. J. Non-Linear Mech. 30 (1995) 391-405.

[4] C. Fetecau and C. Fetecau, Decay of a potential vortex in an Oldroyd-B fluid, Int. J. Eng. Sci. 43 (2005) 340-351.

[5] M. Sajid, Z. Abbas, T. Javed and N. Ali, Boundary layer flow of an Oldroyd-B fluid in the region of a stagnation point over a stretching sheet, Canad. J. Phys. 88 (2010) $635-640$.

[6] L. Zheng, Y. Liu and X. Zhang, Exact solutions for MHD flow of generalized Oldroyd-B fluid due to an infinite accelerating plate, Math. Comput. Model 54 (2011) 780-788. 16.

[7] T. Hayat, S.A. Shehzad, A. Alsaedi and M.S. Alhuthali, Three-dimensional flow of an Oldroyd-B fluid over a surface with convective boundary conditions, Appl. Math. Mech. 34 (2013) 489-500.

[8] S. Abbasbandy, T. Hayat, A. Alsaedi and M. M. Rashidi, Numerical and analytical solutions for Falkner-Skan flow of MHD Oldroyd-B fluid, Int. J. Numer. Meth. Heat \& Fluid Flow 24 (2014) 390-401.

[9] M. Jamil, C. Fetecau, M. Imran, Unsteady helical flows of Oldroyd-B fluids, Commun. Nonlinear Sci. Numer. Simul. 16 (2011) 1378-1386.

[10] C. Fetecau, W. Akhtar, M.A. Imran, D. Vieru, On the oscillating motion of an Oldroyd-B fluid between two infinite circular cylinders, Comput. Math.Appl. 59 (2010) 2836-2845.

[11] T. Hayat, M. Imtiaz, A. Alsaedi and S. Almezal, On Cattaneo-Christov heat flux in MHD flow of Oldroyd-B fluid with homogeneous-heterogeneous reactions, J. Magnet. Magn. Mater. 401 (2016) 296-303.

[12] T. Hayat, M. Waqas, A. Shehzad and A. Alsaedi, On 2D stratified flow of an Oldroyd-B fluid with chemical reaction: An application of non-Fourier heat flux theory, J. Mol. Liq. 223 (2016) 566-571.

[13] T. Hayat, S. Qayyum, A. Alsaedi and M. Waqas, Simultaneous influences of mixed convection and nonlinear thermal radiation in stagnation point flow of Oldroyd-B fluid towards an unsteady convectively heated stretched surface, J. Mol. Liq. 224 (2016) 811-817.

[14] Y. Zhang, M. Zhang and Y. Bai, Flow and heat transfer of an Oldroyd-B nanofluid thin film over an unsteady stretching sheet, J. Mol. Liq. 220 (2016) 665-670.

[15] S U S Choi, Enhancing thermal conductivity of fluids with nanoparticles, ASME Fluids Eng. Division, 231 (1995) 99-105. 
S. Mishra et al.

International Journal of Thermofluid Science and Technology (2020), Volume 7, Issue 1, Paper No. 20070104

[16] Y. Xuan, Q. Li, Investigation on convective heat transfer and flow features of nanofluids, J. Heat Transfer, 125 (2003) 151-155.

[17] J. Buongiorno, Convective Transport in Nanofluids, Journal of Heat Transfer 128 (2006) 241250 .

[18] W. Khan and I. Pop, Boundary-layer flow of a nanofluid past a stretching sheet, Int. J. Heat Mass Transf. 53 (2010) 2477-2483.

[19] T. Hayat, M. Imtiaz and A. Alsaedi, Boundary layer flow of Oldroyd-B fluid by exponentially stretching sheet, Appl. Math. Mech. 37 (2016) 573-582.

[20] I. S. Shivakumara, M. Dhananjaya and C. Ng, Thermal convective instability in an Oldroyd-B nanofluid saturated porous layer, Int. J. Heat Mass Transf. 84 (2015) 167-177.

[21] M K Nayak, MHD 3D flow and heat transfer analysis of nanofluid by shrinking surface inspired by thermal radiation and viscous dissipation, Int. J Mech. Sci. 124-125 (2017) 185193.

[22] M K Nayak, S Shaw, V S Pandey and A J Chamkha, Combined effects of slip and convective boundary condition on MHD 3Dstretched flow of nanofluid through porous media inspired by non-linear thermal radiation, Indian J Phys, doi.org/10.1007/s12648-018-1188-2.

[23] M.K. Nayak, N.S. Akbar, V.S. Pandey, Z.H. Khan, D. Tripathi, 3D free convective MHD flow of nanofluid over permeable linear stretching sheet with thermal radiation, Powder Technol. 315 (2017) 205-215.

[24] S.S. Ghadikolaei, Kh. Hosseinzadeh, D.D. Ganji, M. Hatami, $\mathrm{Fe}_{3} \mathrm{O}_{4}-\left(\mathrm{CH}_{2} \mathrm{OH}\right)_{2}$ nanofluid analysis in a porous medium under MHD radiative boundary layer and dusty fluid, J. Mol. Liq., 258 (2018) 172-185.

[25] T. Hayat, T. Muhammad, S.A. Shehzad and A. Alsaedi, An analytical solution for magnetohydrodynamic Oldroyd-B nanofluid flow induced by a stretching sheet with heat generation/absorption, Int. J. Ther. Sci., 111 (2017) 274-288.

[26] M. Khan, A. Hafeez and J. Ahmed, Impacts of non-linear radiation and activation energy on the axisymmetric rotating flow of Oldroyd-B fluid, Phys. A: Stat. Mech.Appl., (2020) 124085.

[27] B. Mahanthesh, B.J. Gireesha, S.A. Shehzad, F.M. Abbasi and R.S.R. Gorla, Nonlinear threedimensional stretched flow of an Oldroyd-B fluid with convective condition, thermal radiation, and mixed convection, Appl. Math. Mech. Eng. Edi., 38 (2017) 969-98.

[28] K.G. Kumar, G.K. Ramesh, B.J. Gireesha and R.S.R. Gorla, Characteristics of Joule heating and viscous dissipation on three-dimensional flow of Oldroyd B nanofluid with thermal radiation, Alex. Eng. J., 57 (2018) 2139-2149.

[29] M.K. Nayak, Chemical reaction effect on MHD viscoelastic fluid over a stretching sheet through porous medium, Meccanica 51 (2016) 1699-1711.

[30] Y. Zhang, B. Yuan, Y. Bai, Y. Cao, Y. Shen, Unsteady Cattaneo-Christov double diffusion of Oldroyd-B fluid thin film with relaxation-retardation viscous dissipation and relaxation chemical reaction, Powder Technol. DOI: 10.1016/j.powtec.2018.07.049.

[31] M. Mustafa, T. Hayat, A. Alsaedi, "Rotating flow of Oldroyd-B fluid over stretchable surface with Cattaneo-Christov heat flux: analytic solutions", Int J of Num Meth Heat Fluid Flow, DOI: 10.1108/HFF-08-2016-0323.

[32] T. Hayat, S. Qayyum, S. A. Shehzad, A. Alsaedi, Magnetohydrodynamic three-dimensional nonlinear convection flow of Oldroyd-B nanoliquid with heat generation/absorption, DOI: 10.1016/j.molliq.2017.01.045.

[33] M. S. Abel, J. V. Tawade, M. M. Nandeppanavar, MHD flow and heat transfer for the upperconvected Maxwell fluid over a stretching sheet, Meccanica 47 (2012) 385-93.

[34] A. M. Megahed, Variable fluid properties and variable heat flux effects on the flow and heat transfer in a non-Newtonian Maxwell fluid over an unsteady stretching sheet with slip velocity, Chin Phys B 22 (2013) 701-711.

[35] M. Waqas, M. Ijaz Khan, T. Hayat, A. Alsaedi, Stratified flow of an Oldroyd-Bnanoliquid with heat generation, Results in Physics 7 (2017) 2489-2496. 
S. Mishra et al.

International Journal of Thermofluid Science and Technology (2020), Volume 7, Issue 1, Paper No. 20070104 